\title{
RENEWAL FUNCTION ASYMPTOTICS REFINED À LA FELLER
}

BY

\author{
DARYL DALEY (MELBOURNE)
}

Dedicated to Tomasz Rolski, a constant colleague over forty years, distance notwithstanding.

\begin{abstract}
Feller's volume 2 shows how to use the Key Renewal Theorem to prove that in the limit $x \rightarrow \infty$, the renewal function $U(x)$ of a renewal process with nonarithmetic generic lifetime $X$ with finite mean $\mathrm{E}(X)=1 / \lambda$ and second moment differs from its linear asymptote $\lambda x$ by the quantity $\frac{1}{2} \lambda^{2} \mathrm{E}\left(X^{2}\right)$. His first edition (1966) (but not the second in 1971) asserted that a similar approach would refine this asymptotic result when $X$ has finite higher order moments. The paper shows how higher order moments may justify drawing conclusions from a recurrence relation that exploits a general renewal equation and further appeal to the Key Renewal Theorem.
\end{abstract}

2010 AMS Mathematics Subject Classification: Primary: 60K05.

Key words and phrases: Renewal function, asymptotics of refined iterates, moment condition.

\section{INTRODUCTION}

This paper concerns two remarks in the first edition of Volume 2 of Feller's (1966) treatise on probability theory that are missing in the second edition (1971), see [6]. The context is that of the renewal function $U$ of a renewal process whose generic lifetime random variable $X$ has a non-lattice distribution function $F$ whose first two moments are finite (here, $U(x)=\sum_{n=0}^{\infty} F^{n *}(x)$ for $x \geqslant 0$ with $F^{n *}$ the $n$-fold convolution power of $F$ ). Write $\mathrm{E}(X)=1 / \lambda$. After using the Key Renewal Theorem (Feller, 1966, Theorem XI.1.2) to show that

$$
U(x)-\lambda x \rightarrow \frac{1}{2} \lambda^{2} \mathrm{E}\left(X^{2}\right) \quad \text { as } x \rightarrow \infty,
$$

Feller wrote (p. 357) "[this] asymptotic expansion of $U$ may be further refined if $F$ has moments of higher order", and (p. 372) "this method [of using the general renewal equation to establish $(\mathbb{L} . \mathbb{D})]$ can be used for better estimates when higher moments exist." 
Associated with any given renewal process as described above is the general renewal equation

$$
Z(x)=z(x)+\int_{0}^{x} Z(x-u) F(\mathrm{~d} u), \text { so } Z(x)=\int_{0}^{x} z(x-u) U(\mathrm{~d} u),
$$

in which $z$ is the generator for the solution function $Z$. By linearity, if the pairs $\left(Z_{1}, z_{1}\right)$ and $\left(Z_{2}, z_{2}\right)$ satisfy the same general renewal equation with given lifetime d.f. $F$, then so does also the pair $\left(a_{1} Z_{1}-a_{2} Z_{2}, a_{1} z_{1}-a_{2} z_{2}\right)$ for any real finite $a_{1}, a_{2}$, as Feller exploited. In this paper I establish and build on a result asserted in Daley and Mohan [3]. That result enables us to construct a sequence of functions which involve iterated integrals of the renewal function, its asymptote and, where applicable, finiteness of these iterated differences for which the Key Renewal Theorem holds. A necessary and sufficient condition for the $n$-th step in this reasoning is that the lifetime r.v. $X$ should have a finite $(n+1)$-st order moment, as in Feller's first assertion.

It remains unclear to us as to how to exploit the Key Renewal Theorem to obtain "better estimates when higher moments exist" (second quotation from Feller), because detailed asymptotic behaviour of the difference function $Z_{1}(x):=$ $U(x)-\lambda x$ is needed. Stronger properties (limits of analytic functions and the Riemann-Lebesgue theorem) are exploited in Stone [8], based also on Feller and Orey's work [7]. To be fair, this second quotation was made in the (two-sided) random walk setting.

\section{BASIC RESULT AND RECURRENCE RELATIONS}

Our basic result appeared without proof in Daley and Mohan [3] in the setting of a general random walk; we give it here with proof in the simpler case of a renewal process, i.e. on $\mathbb{R}_{+}$.

THEOREM A. Let the real-valued function $Z$ on $\mathbb{R}_{+}$satisfy the general renewal equation (1.2), with generator $z$, in which the lifetime d.f. $F$ of the nonnegative r.v. $X$ has first moment $1 / \lambda$.

(a) When $z$ is directly Riemann integrable and $C=\int_{0}^{\infty} z(u) \mathrm{d} u, Z(x) \rightarrow \lambda C$ $(x \rightarrow \infty)$.

(b) Then $\widetilde{Z}(x):=\int_{0}^{x}[Z(y)-\lambda C] \mathrm{d} y$ satisfies (1.2) with generator

$$
\tilde{z}(x):=\int_{x}^{\infty}[C \lambda \bar{F}(v)-z(v)] \mathrm{d} v,
$$

where $\bar{F}(x)=1-F(x)$.

(c) When $\int_{x}^{\infty} z(v) \mathrm{d} v$ is directly Riemann integrable and $X$ has finite second moment,

$$
\widetilde{Z}(x) \rightarrow \lambda \widetilde{C}:=\lambda \int_{0}^{\infty} \tilde{z}(y) \mathrm{d} y \quad(x \rightarrow \infty),
$$

where $\widetilde{C}=\lambda C \frac{1}{2} \mathrm{E}\left(X^{2}\right)-\int_{0}^{\infty} v z(v) \mathrm{d} v$. 
Proof. The assertion (a) is simply the Key Renewal Theorem (e.g., Theorem XI.1.2 of Feller, 1966).

For (b), the integral representation in (1.2) implies that

$$
\begin{aligned}
\int_{0}^{x} Z(y) \mathrm{d} y & =\int_{0}^{x} \mathrm{~d} y \int_{0}^{y} z(y-u) U(\mathrm{~d} u)=\int_{0}^{x} U(\mathrm{~d} u) \int_{u}^{x} z(y-u) \mathrm{d} y \\
& =\int_{0}^{x} U(\mathrm{~d} u) \int_{0}^{x-u} z(v) \mathrm{d} v=\int_{0}^{x}\left[C-\int_{x-u}^{\infty} z(v) \mathrm{d} v\right] U(\mathrm{~d} u) .
\end{aligned}
$$

Thus, $\int_{0}^{x} Z(u) \mathrm{d} u$ as a solution function of (1.2) has as its generator $\int_{0}^{x} z(u) \mathrm{d} u$, $=C-\int_{x}^{\infty} z(u) \mathrm{d} u$ when $z$ is directly Riemann integrable. $C \lambda x$ as a solution to (1.2) has as its generator the function equal to

$$
\begin{aligned}
C \lambda x-C \int_{0}^{x} \lambda(x-y) F(\mathrm{~d} y) & =C \int_{0}^{x} \lambda[1-F(v)] \mathrm{d} v \\
& =C-C \int_{x}^{\infty} \lambda \bar{F}(v) \mathrm{d} v .
\end{aligned}
$$

Then the generator for the solution function $\widetilde{Z}(x)=\int_{0}^{x} Z(u) \mathrm{d} u-\lambda C x$ is the difference function

$$
\tilde{z}(x)=C-\int_{x}^{\infty} z(v) \mathrm{d} v-C+C \int_{x}^{\infty} \lambda \bar{F}(v) \mathrm{d} v=\int_{x}^{\infty}[C \lambda \bar{F}(v)-z(v)] \mathrm{d} v
$$

For (c), $\mathrm{E}\left(X^{2}\right)<\infty$ implies

$$
\lim _{x \rightarrow \infty} \int_{0}^{x} \mathrm{~d} y \int_{y}^{\infty} \lambda \bar{F}(u) \mathrm{d} u=\lim _{x \rightarrow \infty} \int_{0}^{\infty} \min (x, u) \bar{F}(u) \mathrm{d} u<\infty,
$$

and with direct Riemann integrability of the function $\int_{x}^{\infty} z(v) \mathrm{d} v$ this implies (c) via the Key Renewal Theorem.

In the notation of Theorem A, set

$$
\begin{aligned}
& Z_{1}(x):=Z(x)=U(x)-\lambda x, \\
& z_{1}(x):=z(x)=\int_{x}^{\infty} \lambda \bar{F}(u) \mathrm{d} u, \quad C_{1}:=C=\int_{0}^{\infty} z_{1}(u) \mathrm{d} u,
\end{aligned}
$$

where $C$ is finite. We have

$$
Z_{1}(x)-\lambda C_{1}=\int_{0}^{x} z_{1}(x-u) U(\mathrm{~d} u)-\lambda \int_{0}^{\infty} z_{1}(u) \mathrm{d} u
$$


hence

$$
\begin{aligned}
& \lambda \int_{x}^{\infty} z_{1}(u) \mathrm{d} u-\left[\lim _{y \rightarrow \infty} Z_{1}(y)-Z_{1}(x)\right]=\int_{0}^{x} z_{1}(x-u)[U(\mathrm{~d} u)-\lambda \mathrm{d} u] \\
& \quad=\lambda \int_{0}^{x}[U(\mathrm{~d} u)-\lambda \mathrm{d} u] \int_{x-u}^{\infty} \bar{F}(v) \mathrm{d} v=\lambda \int_{0}^{\infty} \bar{F}(v) \mathrm{d} v \int_{x-v}^{x}[U(\mathrm{~d} u)-\lambda \mathrm{d} u] \\
& \quad=\lambda \int_{0}^{\infty}\left[U(x)-U\left((x-v)_{+}\right)-\lambda \min \{x, v\}\right] \bar{F}(v) \mathrm{d} v .
\end{aligned}
$$

In the last integrand, $U(x)-U\left((x-v)_{+}\right) \leqslant U(v)$ and $0 \leqslant U(v)-\lambda v \leqslant \lambda C_{1}$ for all $v$, so the integrand is uniformly bounded above. Split the range of integration to $(0, V)$ and $(V, \infty)$. Take $V$ sufficiently large that $\int_{V}^{\infty} \bar{F}(v) \mathrm{d} v$ is arbitrarily small positive; then for fixed $V$, take $x$ sufficiently large that $|U(x)-U(x-v)-\lambda v|$ is arbitrarily small positive for all $0<v<V$, by Blackwell's form of the renewal theorem. Then the right-hand side is $o(1)$ as $x \rightarrow \infty$, so

$$
\lambda C_{1}-Z_{1}(x)=\lambda \int_{x}^{\infty} z_{1}(u) \mathrm{d} u+o(1)=\lambda \int_{x}^{\infty}(u-x) \lambda \bar{F}(u) \mathrm{d} u+o(1) \quad(x \rightarrow \infty) .
$$

Now, when $\int_{1}^{\infty} y^{\gamma} \bar{F}(y) \mathrm{d} y<\infty$ for any $\gamma>1, \int_{x}^{\infty} z_{1}(u) \mathrm{d} u$ is of smaller order than $o(1)$ for $x \rightarrow \infty$. In other words, the argument of Theorem A has not (yet) yielded any finer estimate of the asymptotic behaviour of $U(x)-\lambda x$ than the constant $\lambda C_{1}$.

In the setting of Theorem A, define $\left(Z_{2}, z_{2}, C_{2}\right)=(\widetilde{Z}, \tilde{z}, \widetilde{C})(\widetilde{C}$ need not be finite). More generally, for integers $n=2,3, \ldots$, let $(Z, z, C)=\left(Z_{n}, z_{n}, C_{n}\right)$ and, provided $C_{n}$ is finite, define $\left(Z_{n+1}, z_{n+1}, C_{n+1}\right)=(\widetilde{Z}, \tilde{z}, \widetilde{C})$ as in Theorem A (here $C_{n+1}=\widetilde{C}$ need not be finite). It now follows that when $\left\{\left(Z_{j}, z_{j}\right): j=\right.$ $1, \ldots, n\}$ are well defined with $\left\{C_{1}, \ldots, C_{n}\right\}$ finite, the following recurrence relations hold for $j=1, \ldots, n$ :

$$
\begin{aligned}
& z_{j+1}(x)=C_{j} \int_{x}^{\infty} \lambda \bar{F}(u) \mathrm{d} u-\int_{x}^{\infty} z_{j}(u) \mathrm{d} u \\
& Z_{j+1}(x)=\int_{0}^{x}\left[Z_{j}(v)-\lambda C_{j}\right] \mathrm{d} v=\int_{0}^{x} \tilde{z}_{j+1}(x-u) U(\mathrm{~d} u) .
\end{aligned}
$$

In (2.5), when $\mathrm{E}\left(X^{2}\right)$ is finite, $\widetilde{C}_{j+1}$ is finite if and only if $\int_{0}^{\infty} \mathrm{d} x \int_{x}^{\infty} z_{j}(u) \mathrm{d} u=$ $\int_{0}^{\infty} u z_{j}(u) \mathrm{d} u$ is well defined and finite. For $j=1$, using (2.3), it follows that $\int_{0}^{\infty} u z_{1}(u) \mathrm{d} u=\int_{0}^{\infty} \frac{1}{2} v^{2} \lambda \bar{F}(v) \mathrm{d} v$, which is finite if and only if $\mathrm{E}\left(X^{3}\right)<\infty$. Applying the Key Renewal Theorem to the second equality in (2.6) yields the following. 
COROLlary 2.1. Let $m_{j}=\mathrm{E}\left(X^{j}\right), j=1,2, \ldots$ For $m_{2}<\infty$, whether $\mathrm{E}\left(X^{3}\right)$ is finite or infinite, we have

$$
\begin{aligned}
\lambda C_{2} & :=\lim _{x \rightarrow \infty} \int_{0}^{x}\left[U(y)-\lambda y-\frac{1}{2} \lambda^{2} \mathrm{E}\left(X^{2}\right)\right] \mathrm{d} y \\
& =\left(\frac{1}{2} \lambda \mathrm{E}\left(X^{2}\right)\right)^{2}-\frac{1}{6} \lambda^{2} \mathrm{E}\left(X^{3}\right)=\lambda C_{1} \frac{m_{2}}{2 !}-\frac{\lambda m_{3}}{3 !} .
\end{aligned}
$$

Return to (2.5) but now take $j=2$. Let us assume $\mathrm{E}\left(X^{3}\right)<\infty$. Then $C_{2}$ is finite and $\int_{0}^{\infty} z_{3}(x) \mathrm{d} x$ is well defined because the integral over $\mathbb{R}_{+}$of the first term on the right-hand side is necessarily finite. Then $C_{3}$ is finite if and only if $\int_{0}^{\infty} u z_{2}(u) \mathrm{d} u$ is finite; substitution for $z_{2}$ from (2.5) shows this to occur if and only if $\int_{0}^{\infty} \mathrm{d} x \int_{x}^{\infty} u \mathrm{~d} u \int_{u}^{\infty} \lambda \bar{F}(v) \mathrm{d} v$ is finite, i.e. because the integrand is nonnegative, if and only if $\int_{0}^{\infty} v^{3} \bar{F}(v) \mathrm{d} v<\infty$, i.e. $\mathrm{E}\left(X^{4}\right)<\infty$.

This argument can be continued for as long as $\mathrm{E}\left(X^{j}\right)$ is finite, justifying the next result.

COROLlary 2.2. When $C_{n-1}$ is finite, $C_{n}$ is well defined; $C_{n}$ is finite if and only if $\mathrm{E}\left(X^{n+1}\right)$ is finite.

A more transparent proof of Corollary 2.2 proceeds via a chain of substitutions exploiting (2.5) as follows. Assume $C_{n}$ is finite. Then from (2.5) with, successively, $j=n, n-1, \ldots, 1$, we see that $z_{n+1}(x)$ equals

$$
\begin{aligned}
& C_{n} \int_{x}^{\infty} \lambda \bar{F}(u) \mathrm{d} u-C_{n-1} \int_{x}^{\infty} \mathrm{d} u \int_{u}^{\infty} \lambda \bar{F}(v) \mathrm{d} v+\int_{x}^{\infty} \mathrm{d} u \int_{u}^{\infty} z_{n-1}(v) \mathrm{d} v \\
= & C_{n} \int_{x}^{\infty} \lambda \bar{F}(u) \mathrm{d} u-C_{n-1} \int_{x}^{\infty}(u-x) \lambda \bar{F}(u) \mathrm{d} u+\int_{x}^{\infty}(u-x) z_{n-1}(u) \mathrm{d} u \\
= & C_{n} \int_{x}^{\infty} \lambda \bar{F}(u) \mathrm{d} u-C_{n-1} \int_{x}^{\infty}(u-x) \lambda \bar{F}(u) \mathrm{d} u \\
& +C_{n-2} \int_{x}^{\infty} \frac{(u-x)^{2}}{2 !} \lambda \bar{F}(u) \mathrm{d} u-\int_{x}^{\infty} \frac{(u-x)^{2}}{2 !} z_{n-2}(u) \mathrm{d} u \\
= & \int_{x}^{\infty}\left[\sum_{i=0}^{r} \frac{C_{n-i}(-1)^{i}(u-x)^{i}}{i !}\right] \lambda \bar{F}(u) \mathrm{d} u-(-)^{r} \int_{x}^{\infty} \frac{(u-x)^{r}}{r !} z_{n-r}(u) \mathrm{d} u,
\end{aligned}
$$

which for $r=1, \ldots, n-1$ is equal to

$$
\text { 8) } \begin{aligned}
& \int_{x}^{\infty}\left[\sum_{i=0}^{n-1} \frac{C_{n-i}(-1)^{i}(u-x)^{i}}{i !}\right] \lambda \bar{F}(u) \mathrm{d} u+(-)^{n} \int_{x}^{\infty} \frac{(u-x)^{n-1}}{(n-1) !} z_{1}(u) \mathrm{d} u \\
= & \int_{x}^{\infty}\left[\sum_{i=0}^{n-1} \frac{C_{n-i}(-1)^{i}(u-x)^{i}}{i !}\right] \lambda \bar{F}(u) \mathrm{d} u+(-)^{n} \int_{x}^{\infty} \frac{(v-x)^{n}}{n !} \lambda \bar{F}(v) \mathrm{d} v .
\end{aligned}
$$


All the integrals in (2.8) are finite if and only if $n ! m_{n+1}=\int_{0}^{\infty} v^{n} \bar{F}(v) \mathrm{d} v<\infty$ as in Corollary 2.2. When this condition is met, we can set $x=0$ in (2.8) and obtain the next result; (2.8) is the simplest non-trivial case of (2.9) below.

TheOREM 2.1. When $m_{n+1}$ is finite, $\lim _{x \rightarrow \infty} Z_{n}(x)=\lambda C_{n}$ finite, and $\left\{C_{j}\right.$ : $j=1, \ldots, n\}$ satisfy

$$
C_{r}=\sum_{i=1}^{r-1}(-1)^{i+1} C_{r-i} \frac{m_{i+1}}{(i+1) !}+(-1)^{r+1} \frac{m_{r+1}}{(r+1) !} \quad(r=1,2, \ldots, n) .
$$

Corollary 2.2 shows that the sequence of functions $\left\{Z_{n}\right\}$ is finite only for as many moments of the generic r.v. $X$ are finite. This is some amplification of Feller's first remark.

For Feller's other remark, suppose $m_{r+1}<\infty$ for some integer $r>n$ in (2.8); defining $C_{0}=1$, rewrite (2.8) as

$$
z_{n+1}(x)=\sum_{i=0}^{n} \int_{x}^{\infty} \frac{C_{n-i}(-1)^{i}}{i !}(u-x)_{+}^{i} \lambda \bar{F}(u) \mathrm{d} u .
$$

The finiteness of $m_{r+1}$ implies that the magnitude of the $i$-th integral here is bounded above by $\lambda\left|C_{n-i}\right| / i$ ! times

$$
\int_{x}^{\infty} \frac{1}{u^{r-i}}\left(1-\frac{x}{u}\right)^{i} u^{r} \bar{F}(u) \mathrm{d} u<\frac{1}{x^{r-i}} \int_{x}^{\infty} u^{r} \bar{F}(u) \mathrm{d} u .
$$

Since the last integral is $o(1)$ for $x \rightarrow \infty$, uniformly in $i=0,1, \ldots, n$, we can conclude that $x^{r-n} z_{n+1}(x)=o(1)$ for $x \rightarrow \infty$.

In terms of explaining Feller's second remark about "better estimates of the renewal function when higher moments exist", this property is the best we have been able to find. Manipulations similar to (2.11) in (2.8) did not lead to any recognizable series in powers of $1 / x$ analogous to standard Taylor series expansion. Further, because the key relations (2.5) and (2.6) are recursive in nature, only integer powers are involved and results as powerful as Stone [8] obtained using Fourier methods do not appear to be accessible by Daley and Mohan's approach [3].

\section{ANOTHER RECURRENCE RELATION}

Using (2.6) as a basis of recurrence relations yields the following expansion:

$$
\begin{aligned}
Z_{n+1}(x) & =\int_{0}^{x}\left[Z_{n}(u)-\lambda C_{n}\right] \mathrm{d} u \\
& =-\lambda C_{n} x+\int_{0}^{x} \mathrm{~d} u \int_{0}^{u}\left[Z_{n-1}(v)-\lambda C_{n-1}\right] \mathrm{d} v \\
& =-\lambda C_{n} x-\lambda C_{n-1} \frac{x^{2}}{2}+\int_{0}^{x}(x-v) Z_{n-1}(v) \mathrm{d} v \\
& =-\sum_{i=0}^{n-1} \lambda C_{n-i} \frac{x^{i+1}}{(i+1) !}+\int_{0}^{x} \frac{(x-v)^{n-1}}{(n-1) !} Z_{1}(v) \mathrm{d} v,
\end{aligned}
$$


and

$$
\begin{aligned}
\int_{0}^{x} \frac{(x-u)^{n-1}}{(n-1) !} Z_{1}(u) \mathrm{d} u & =x^{n} \int_{0}^{x} \frac{1}{(n-1) !}\left(1-\frac{u}{x}\right)^{n-1} Z_{1}(u) \frac{\mathrm{d} u}{x} \\
& =x^{n} \frac{\lambda C_{1}}{n !}[1+o(1)] .
\end{aligned}
$$

The term $i=n-1$ in the sum occurring in the last equality of (3.1) equals $-\lambda C_{1} x^{n} / n$ !, which is the negative of the dominant term in (3.2), so

$$
Z_{n+1}(x)+\sum_{i=0}^{n-2} \lambda C_{n-i} \frac{x^{i+1}}{(i+1) !}=o\left(x^{n}\right) .
$$

However, for large $x$, the right-hand side dominates all terms on the left-hand side (when $C_{n+1}$ is finite), so the expansion in (3.1) does not add information in the sense of Feller's comments.

Equivalently, we have

$$
\begin{aligned}
Z_{n+1}(x)+\sum_{i=0}^{n-1} \lambda C_{n-i} \frac{x^{i+1}}{(i+1) !} & =\int_{0}^{x} \frac{(x-v)^{n-1}}{(n-1) !} \mathrm{d} v \int_{0}^{v} z_{1}(v-u) U(\mathrm{~d} u) \\
& =\int_{0}^{x} \frac{w^{n-1}}{(n-1) !} \mathrm{d} w \int_{0}^{x-w} z_{1}(x-w-u) U(\mathrm{~d} u) \\
& =\int_{0}^{x} U(\mathrm{~d} u) \int_{0}^{x-u} \frac{w^{n-1}}{(n-1) !} z_{1}(x-u-w) \mathrm{d} w .
\end{aligned}
$$

\section{FURTHER RESULTS}

The referee has drawn my attention to older work by Carlsson [2] and more recent papers by Blanchet and Glynn [1], and Dombry and Rabehasaina [5] all concerned with asymptotic expansions of the renewal function $U$. Carlsson's analysis exploits properties of the Fourier transform $f(t)=\mathrm{E}\left(e^{i t X}\right)$, notably of $1-f(t)$ and expansions ( $t$ is real). Blanchet and Glynn apply Carlsson's results to the particular problem of random geometric sums. I have not seen Dombry and Rabehasaina's work. Carlsson's study includes examples of lifetime distributions $F$ that are weakly nonlattice, i.e. of d.f.s that are nonarithmetic but differ from a lattice distribution only by a "very small amount".

\section{REFERENCES}

[1] J. Blanchet and P. Glynn, Uniform renewal theory with applications to expansions of random geometric sums, Adv. in Appl. Probab. 39 (2007), pp. 1070-1097.

[2] H. Carlsson, Remainder term estimates of the renewal function, Ann. Probab. 11 (1983), pp. $123-157$. 
[3] D. J. Daley and N. R. Mohan, Asymptotic behaviour of the variance of renewal processes and random walks, Ann. Probab. 6 (1978), pp. 516-521.

[4] D. J. Daley and D. Vere-Jones, An Introduction to the Theory of Point Processes. Volume I: Elementary Theory and Methods, second edition, Springer, New York 2003.

[5] C. Dombry and L. Rabehasaina, Asymptotic expansion for renewal functions and applications to ruin theory, arXiv, 2016.

[6] W. Feller, An Introduction to the Theory of Probability and Its Applications, vol. 2, second edition, Wiley, New York 1971.

[7] W. Feller and S. Orey, A renewal theorem, J. Math. Mech. 10 (1961), pp. 619-624.

[8] C. Stone, On characteristic functions and renewal theory, Trans. Amer. Math. Soc. 120 (1965), pp. 327-342.

\section{Daryl Daley}

Department of Mathematics and Statistics

The University of Melbourne

Victoria 3010, Melbourne, Australia

E-mail:dndaley@gmail.com 